Ophthalmologe $2017 \cdot 114: 77-78$ DOI 10.1007/s00347-016-0429-z Online publiziert: 10. Januar 2017

o) Springer Medizin Verlag Berlin 2016

CrossMark

V. Prokosch · D. C. Dragnea - S. Pitz

Augenklinik der Universitätsmedizin, Johannes Gutenberg UniversitätMainz, Mainz, Deutschland

\title{
Erratum zu: Differenzialdiagnose der Papillenschwellung. Wichtige Differenzialdiagnosen im Überblick
}

\section{Erratum zu:}

Ophthalmologe (2016) 113:967-981

DOI 10.1007/s00347-016-0353-2

In diesem Beitrag der Rubrik „CME Zertifizierte Fortbildung" wurde eine Frage nicht korrekt wiedergegeben. Wir bitten, die korrekte Version des CMEFragebogens in dem Online-Kurs zu berücksichtigen.

Bei Verdacht einer intraorbitalen Raumforderung mit Papillenschwellung ist welches bildgebende Verfahren das Verfahren der Wahl:

Bulbussonographie

Computertomographie

$\square$ Farbkodierte Duplexsonographie der Orbita

$\square$ Magnetresonanztomographie (MRT)

$\square$ Optische Kohärenztomographie (OCT)

\section{Korrespondenzadresse}

PD Dr. V. Prokosch

Augenklinik der Universitätsmedizin, Johannes Gutenberg UniversitätMainz Langenbeckstr. 1, 55131 Mainz, Deutschland verena.prokosch-willing@unimedizinmainz.de 
Hier steht eine Anzeige.

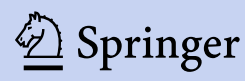

